\title{
LONG-RANGE DEPENDENCE OF MARKOV CHAINS IN DISCRETE TIME ON COUNTABLE STATE SPACE
}

\author{
K. J. E. CARPIO* AND \\ D. J. DALEY, ${ }^{* * *}$ The Australian National University
}

\begin{abstract}
When $\left\{X_{n}\right\}$ is an irreducible, stationary, aperiodic Markov chain on the countable state space $X=\{i, j, \ldots\}$, the study of long-range dependence of any square integrable functional $\left\{Y_{n}\right\}:=\left\{y_{X_{n}}\right\}$ of the chain, for any real-valued function $\left\{y_{i}: i \in \mathcal{X}\right\}$, involves in an essential manner the functions $Q_{i j}^{n}=\sum_{r=1}^{n}\left(p_{i j}^{r}-\pi_{j}\right)$, where $p_{i j}^{r}=\mathrm{P}\left\{X_{r}=\right.$ $\left.j \mid X_{0}=i\right\}$ is the $r$-step transition probability for the chain and $\left\{\pi_{i}: i \in \mathcal{X}\right\}=\mathrm{P}\left\{X_{n}=i\right\}$ is the stationary distribution for $\left\{X_{n}\right\}$. The simplest functional arises when $Y_{n}$ is the indicator sequence for visits to some particular state $i, I_{n i}=I_{\left\{X_{n}=i\right\}}$ say, in which case $\lim \sup _{n \rightarrow \infty} n^{-1} \operatorname{var}\left(Y_{1}+\cdots+Y_{n}\right)=\lim \sup _{n \rightarrow \infty} n^{-1} \operatorname{var}\left(N_{i}(0, n]\right)=\infty$ if and only if the generic return time random variable $T_{i i}$ for the chain to return to state $i$ starting from $i$ has infinite second moment (here, $N_{i}(0, n]$ denotes the number of visits of $X_{r}$ to state $i$ in the time epochs $\{1, \ldots, n\})$. This condition is equivalent to $Q_{j i}^{n} \rightarrow \infty$ for one (and then every) state $j$, or to $\mathrm{E}\left(T_{j j}^{2}\right)=\infty$ for one (and then every) state $j$, and when it holds, $\left(Q_{i j}^{n} / \pi_{j}\right) /\left(Q_{k k}^{n} / \pi_{k}\right) \rightarrow 1$ for $n \rightarrow \infty$ for any triplet of states $i, j, k$.
\end{abstract}

Keywords: Markov chain; long-range dependence; Hurst index; moment index; functional 2000 Mathematics Subject Classification: Primary 60J10

\section{Introduction and main results}

A real-valued stationary stochastic process $\left\{Y_{n}\right\}$ with finite second moment is long-range dependent when the asymptotic second-order behaviour of

$$
\operatorname{var}\left(Y_{1}+\cdots+Y_{n}\right)
$$

exceeds $O(n)$ for large $n$; the 'standard' behaviour, i.e. that (1) equals at most $O(n)$, holds when the components are independent or, for example, satisfy

$$
\sum_{n=1}^{\infty}\left|\operatorname{cov}\left(Y_{0}, Y_{n}\right)\right|<\infty .
$$

In this paper we discuss aspects of real-valued processes that are defined on discrete-time stationary Markov processes $\left\{X_{n}\right\}$ on the countable state space $\mathcal{X}=\{i, j, \ldots\}$, and have stationary one-step transition probabilities

$$
p_{i j}=\mathrm{P}\left\{X_{n+1}=j \mid X_{n}=i\right\} \quad \text { for all } n \in \mathbb{N} .
$$

Received 19 June 2006; revision received 5 September 2007.

* Postal address: Centre for Mathematics and its Applications, The Australian National University, Canberra, ACT 0200, Australia.

** Email address: daryl@maths.anu.edu.au 
We assume that the Markov chain is irreducible and positive recurrent, and to simplify some aspects of the discussion, without substantial loss of generality, we assume that the chain is aperiodic. In what follows we freely refer to Chung for various results, meaning Part I of Chung (1967) (and, it should be noted, some of the results that we use are not in the earlier (1960) edition of the book).

The stationarity of $\left\{X_{n}\right\}$ is described via the distribution

$$
\pi_{i}=\mathrm{P}\left\{X_{n}=i\right\} \quad \text { for all } n, i \in \mathcal{X} ;
$$

the $n$-step transition probabilities are denoted by

$$
p_{i j}^{n}=\mathrm{P}\left\{X_{n+r}=j \mid X_{r}=i\right\} \text { for all } r, \text { all } i, j \in \mathcal{X}, n=0,1, \ldots,
$$

so $p_{i j}^{1}=p_{i j}$, and

$$
p_{i j}^{0}= \begin{cases}1 & \text { if } j=i \\ 0 & \text { if } j \neq i\end{cases}
$$

The most general structure for $Y_{n}$, consistent with the Markov setting we have described, is that $Y_{n}=y_{X_{n}}$ for some real-valued set $\left\{y_{i}: i \in \mathcal{X}\right\}$ such that $\sum_{i \in \mathcal{X}} \pi_{i}\left(y_{i}\right)^{2}<\infty$ (e.g. Chung (1967, Sections I.14-16)). For such a structure, we can then readily write down

$$
\operatorname{cov}\left(Y_{0}, Y_{n}\right)=\sum_{i \in \mathcal{X}} \sum_{j \in \mathcal{X}} y_{i} y_{j} \pi_{i}\left(p_{i j}^{n}-\pi_{j}\right), \quad n=0,1, \ldots
$$

Within this form, the simplest functional $\left\{Y_{n}\right\}$ arises when $y_{i}=1$ for some specified state $i$ and $y_{j}=0$ for all other states $j$. Writing $I_{n i}=Y_{n}$ for the realization at time $n$ of this functional, (2) reduces to

$$
\operatorname{cov}\left(I_{0 i}, I_{n i}\right)=\pi_{i}\left(p_{i i}^{n}-\pi_{i}\right),
$$

and the argument of the variance at (1) is just the number of visits of the Markov chain $\left\{X_{n}\right\}$ to state $i$ during the time epochs $1, \ldots, n, N_{i}(0, n]$ say. Then, in this case, (1) becomes $\operatorname{var}\left(N_{i}(0, n]\right)$, and the defining condition of long-range dependence of this particular functional $\left\{I_{n i}\right\}$, namely that

$$
\limsup _{n \rightarrow \infty} \frac{\operatorname{var}\left(N_{i}(0, n]\right)}{n}=\infty,
$$

thus coincides with the definition in Daley and Vesilo (1997) of the long-range dependence of a stationary point process on $\mathbb{R}$. Now since $N_{i}(\cdot)$ is the counting function of a stationary renewal process (for the epochs of visits to the state of a recurrent Markov chain constitute a discrete-time regenerative phenomenon or renewal process), the variance in (3) is expressible as an integral of the associated renewal function or, since the process is in discrete time, as a sum, namely

$$
\begin{aligned}
\operatorname{var}\left(N_{i}(0, n]\right) & =n \pi_{i}\left(1-\pi_{i}\right)+2 \pi_{i} \sum_{r=1}^{n-1} \sum_{s=1}^{n-r}\left(p_{i i}^{s}-\pi_{i}\right) \\
& =-n \pi_{i}\left(1-\pi_{i}\right)+2 \pi_{i} \sum_{r=1}^{n} \sum_{s=0}^{n-r}\left(p_{i i}^{s}-\pi_{i}\right) .
\end{aligned}
$$

The inner sum in (4) equals $\mathrm{E}\left[N_{i}[0, n-r] \mid X_{0}=i\right]-(n+1-r) \pi_{i}$; this expectation is a renewal function for which subadditivity in $n \geq 0$ is a known standard property (e.g. Daley (1978, Equation (4.1))), so it is bounded below by $(n+1-r) \pi_{i}=(n+1-r) \lim _{s \rightarrow \infty} \mathrm{E}\left[N_{i}(0, s]\right] / s$ 
(e.g. Daley and Vere-Jones (2003, Lemma 3.6.I)) and, therefore, these inner sums are nonnegative. Because the renewal process is aperiodic, these sums either converge to a finite limit for large $n-r$, or else diverge to $\infty$; the latter event occurs if and only if the generic random variable of return times from state $i$ back to itself, $T_{i i}$ say, has infinite second moment. In the former case, by a standard result due to Feller (see Theorem 6.5 of Chung (1967), or else, e.g. Equation(12.2) of Feller (1968)),

$$
\begin{aligned}
\lim _{n \rightarrow \infty} \frac{\operatorname{var}\left(N_{i}(0, n]\right)+n \pi_{i}\left(1-\pi_{i}\right)}{2 n} & =\pi_{i} \sum_{n=0}^{\infty}\left(p_{i i}^{n}-\pi_{i}\right) \\
& =\frac{\mathrm{E}\left[T_{i i}\left(T_{i i}-1\right)\right]}{2\left(\mathrm{E}\left(T_{i i}\right)\right)^{2}} \\
& =\frac{1}{2}\left(\pi_{i}\right)^{2}\left(m_{i i}^{(2)}-m_{i i}\right),
\end{aligned}
$$

using notation from Chung (1967) for the first and second moments of $T_{i i}$. We summarise the discussion thus far as below.

Lemma 1. The counting process $\left\{N_{i}(0, n]: n=1,2, \ldots\right\}$ of visits to state $i$ of a stationary, positive recurrent, irreducible, aperiodic Markov chain is long-range dependent if and only if the return time random variable $(R V)$

$$
T_{i i}=\inf \left\{n \geq 1: X_{n}=i \mid X_{0}=i\right\},
$$

has infinite second moment.

By Corollary 1 of Theorem 11.1 from Chung (1967) it follows that $T_{i i}$ has infinite second moment if and only if $T_{j j}$ has infinite second moment for every state $j$, which occurs if and only if, for one such state of the chain (and then all states),

$$
Q_{j j}^{n}=\sum_{r=1}^{n}\left(p_{j j}^{r}-\pi_{j}\right) \rightarrow \infty \quad \text { as } n \rightarrow \infty .
$$

Recall that when the state space $\mathcal{X}$ is finite, the $n$-step transition probabilities $p_{i j}^{n}$ converge to their limits $\pi_{j}$ geometrically fast, in which case sums as in (5) remain bounded as $n$ tends to $\infty$. Lemma 2, below, is now a consequence of negation.

Lemma 2. Long-range dependence behaviour in a countable state space Markov chain is possible only when the state space $\mathcal{X}$ is infinite.

In the next section we study the asymptotic behaviour of the sums

$$
Q_{i j}^{n}=\sum_{r=1}^{n}\left(p_{i j}^{r}-\pi_{j}\right), \quad i, j \in \mathcal{X}, n=1,2, \ldots,
$$

as well as the sums

$$
Q_{i A}^{n}=\sum_{r=1}^{n}\left(p_{i A}^{r}-\pi_{A}\right)=\sum_{r=1}^{n} \sum_{j \in A}\left(p_{i j}^{r}-\pi_{j}\right)=\sum_{j \in A} Q_{i j}^{n},
$$

where, for an arbitrary subset $A \subseteq \mathcal{X}$, both $p_{i A}^{r}=\sum_{j \in A} p_{i j}^{r}$ and $\pi_{A}=\sum_{j \in A} \pi_{j}$ are well defined because they are absolutely convergent sums of their components. 


\section{Long-range dependence as a rate of convergence}

The essence of (5) is twofold. First, as stated there, because long-range dependence of visits to any state $j$ is a property held by either no state or every state in an irreducible Markov chain, it is a class property as described in Section 3 of Chung (1967), so that the term long-range dependence, when applicable, may properly be used to describe the Markov chain rather than merely any particular state. Secondly, the long-range dependence of a state $j$ is a consequence of the 'slow' rate of convergence of the $n$-step transition probability $p_{j j}^{n}$ to their limit $\pi_{j}$. As we now show, this rate of growth of $Q_{i j}^{n}$ is the same for all states $i, j$, and $k$ in the state space; thus, Lemma 3 expresses a refinement of long-range dependence as a class property, exhibiting it in the context of irreducible Markov chains as a rate of convergence property of the transition probabilities. In Lemma 3, only transition probabilities and first-order ergodic quantities like $\pi_{j}$ appear, whereas when the second moments $m_{i i}^{(2)}$ are finite, they would be involved in the limits in the last ratio in (8) (see the display before Lemma 1).

Lemma 3. Let $\mathrm{E}\left(T_{i i}^{2}\right) \equiv m_{i i}^{(2)}=\infty$ for some (and then every) $i \in \mathcal{X}$. For every triple of states $i, j, k$, which need not all be distinct,

$$
\lim _{n \rightarrow \infty} \frac{\left(Q_{i j}^{1}+\cdots+Q_{i j}^{n}\right) / \pi_{j}}{\left(Q_{k k}^{1}+\cdots+Q_{k k}^{n}\right) / \pi_{k}}=\lim _{n \rightarrow \infty} \frac{Q_{i j}^{n} / \pi_{j}}{Q_{k k}^{n} / \pi_{k}}=\lim _{n \rightarrow \infty} \frac{\left(\sum_{r=1}^{n}\left(p_{i j}^{r}-\pi_{j}\right)\right) / \pi_{j}}{\left(\sum_{r=1}^{n}\left(p_{k k}^{r}-\pi_{k}\right)\right) / \pi_{k}}=1 .
$$

Remark. The proof of Lemma 3, below, is based on the results given in Section 11 of Chung (1967). Alternatively, Lemma 3 can be established from first principles by exploiting first-passage and last-exit decomposition results involving associated taboo probabilities ${ }_{i} p_{j k}^{n}=\mathrm{P}\left\{X_{n}=k, X_{r} \neq i(r=1, \ldots, n-1) \mid X_{0}=j\right\}$ and their sums ${ }_{i} p_{j k}^{*}=\sum_{n=1}^{\infty} p_{j k}^{n}$.

Proof of Lemma 3. The second equality, given the third, is simply a matter of definition, and the first equality is a direct consequence of the second because whenever real sequences $\left\{a_{n}\right\}$ and $\left\{b_{n}\right\}$ satisfy $a_{n} \rightarrow \infty$ and $b_{n} / a_{n} \rightarrow 1$ as $n \rightarrow \infty$, then $\left(b_{1}+\cdots+b_{n}\right) /\left(a_{1}+\cdots+a_{n}\right) \rightarrow 1$ also.

Equation (11.19) of Chung (1967) can be restated in the form

$$
0 \leq \sum_{r=0}^{n}\left(p_{k k}^{r}-\pi_{k}\right)-\frac{\pi_{k}}{\pi_{j}} \sum_{r=0}^{n}\left(p_{k j}^{r}-\pi_{j}\right) \leq \sum_{r=0}^{n}{ }_{j} p_{k k}^{r} \leq 1+{ }_{j} p_{k k}^{*}<\infty,
$$

where ${ }_{j} p_{k k}^{*}$ is finite by Theorem 9.3 of Chung (1967). The case $i=k$ of (8) follows directly from (9).

Equation (9) coupled with the fact that $Q_{i i}^{n} \rightarrow \infty$ for one state $i$ if and only if it holds for every state $i$, then shows that $Q_{i j}^{n} \rightarrow \infty$ for every pair of states $i, j$.

Theorem 11.4 of Chung (1967) can be restated in terms of the mean first-passage times $m_{i j}=\mathrm{E}\left(T_{i j}\right), i, j \in \mathcal{X}$, in the form

$$
\lim _{n \rightarrow \infty}\left(\sum_{r=1}^{n}\left(p_{i k}^{r}-\pi_{k}\right)-\sum_{r=1}^{n}\left(p_{j k}^{r}-\pi_{k}\right)\right)=\pi_{k}\left(m_{j k}-m_{i k}\right),
$$

and we may take $j=k$, from which the case $j=k$ of (8) follows.

The remaining case of (8) (i.e. all $i, j$, and $k$ distinct) follows from (9) coupled with the case of (10) in which $j$ and $k$ are interchanged. 
Equation (9) incorporates the dominance property,

$$
\frac{\sum_{r=0}^{n}\left(p_{k j}^{r}-\pi_{j}\right)}{\sum_{r=0}^{n}\left(p_{k k}^{r}-\pi_{k}\right)} \leq \frac{\pi_{j}}{\pi_{k}}=\text { limit as } n \rightarrow \infty \text { of left-hand side; }
$$

it can be rewritten in the form

$$
\frac{Q_{k j}^{n}}{\pi_{j}} \leq \frac{1+Q_{k k}^{n}}{\pi_{k}}
$$

and therefore, for any nonempty subset $A$ of states, using the absolute convergence noted below (7),

$$
\frac{\sum_{r=1}^{n}\left(p_{k A}^{r}-\pi_{A}\right)}{\pi_{A}}=\frac{Q_{k A}^{n}}{\pi_{A}} \leq \frac{1+Q_{k k}^{n}}{\pi_{k}} .
$$

At this point it is tempting to conclude that $Q_{k A}^{n} / Q_{k k}^{n} \rightarrow \pi_{A} / \pi_{k}$ as $n \rightarrow \infty$, but setting $A=\mathcal{X}$ shows that the result of this limit operation can be 0 ; inspection of the argument shows that it is impossible for all the individual terms $Q_{k j}^{n} / Q_{k k}^{n}$ to be nonnegative simultaneously because $Q_{k x}^{n}=\sum_{r=1}^{n}\left(p_{k x}^{r}-\pi_{x}\right)=\sum_{r=1}^{n}(1-1)=0$, so

$$
Q_{k, X \backslash\{k\}}^{n}=-Q_{k k}^{n} \rightarrow-\infty \quad \text { as } n \rightarrow \infty,
$$

and the nonnegativity condition required in a standard application of the dominated convergence theorem is violated. Nevertheless, it easily follows that (see Orey (1961) or Feller (1966))

$$
\left|Q_{k A}^{n}\right| \leq \min \left(\pi_{A}, 1-\pi_{A}\right) \frac{1+Q_{k k}^{n}}{\pi_{k}} \text { for all } k \in X, A \subseteq X
$$

Let $\kappa_{i}$ denote the moment index of the return time RV $T_{i i}$, i.e.

$$
\kappa_{i}=\sup \left\{\alpha: \mathrm{E}\left(T_{i i}^{\alpha}\right)<\infty\right\},
$$

so, for an irreducible stationary Markov chain, $\kappa_{i}$ is independent of the state $i$ by Theorem 11.1 of Chung (1967), and we can write $\kappa=\kappa_{i}($ all $i \in \mathcal{X}$ ) for the moment index of the return times of $\left\{X_{n}\right\}$. It then follows, from Daley (1999), that when $\left\{X_{n}\right\}$ is long-range dependent, the Hurst index of each and every process $\left\{I_{n i}\right\}$ equals $\frac{1}{2}(3-\kappa) \equiv H$ say, and

$$
H=\inf \left\{h: \limsup _{n \rightarrow \infty} \frac{\operatorname{var}\left(N_{i}(0, n]\right)}{n^{2 h}}<\infty\right\} \text { for all } i .
$$

Indeed, from Lemma 3 it follows that the rate of growth of the numerator here is a class property, and from Sgibnev (1981) we know that

$$
\begin{aligned}
Q_{k k}^{n}+1-\pi_{k} & =\sum_{r=0}^{n}\left(p_{k k}^{r}-\pi_{k}\right) \sim\left(\pi_{k}\right)^{2} \sum_{r=1}^{\infty} \min (r, n) \mathrm{P}\left\{T_{k k}>r\right\} \\
& =\left(\pi_{k}\right)^{2} \sum_{r=1}^{\infty} \min (r, n) \sum_{s=r+1}^{\infty} f_{k k}^{s},
\end{aligned}
$$

where $f_{k k}^{s}={ }_{k} p_{k k}^{s}=\mathrm{P}\left\{T_{k k}=s\right\}$ is the first-return distribution for the state $k$ (Sgibnev's result is quoted as Exercise 4.4.5(c) of Daley and Vere-Jones (2003)). 
Sgibnev (1996) has a further result, more general than Lemma 3, that is a solidarity result for the times between visits to a state in a Markov renewal process. First, recall that a discrete-time Markov chain like $\left\{X_{n}\right\}$, as we are considering, is a special case of a Markov renewal process in which the sojourn time in each and every state is the same, and equal to the unit of time. In a general irreducible, positive recurrent Markov renewal process (for which each state therefore has finite mean sojourn time), these sojourn times may vary, and the long-range dependence in such a Markov renewal process can arise from either sojourn times in some state having infinite second moment, or else recurrence times between visits to a given state having infinite second moment (indeed, this latter case is necessary for long-range dependence). This implies that in the Markov chains we are considering, long-range dependence can arise only via the latter mechanism.

Sgibnev's method for deducing his long-range dependent solidarity property is similar to the manipulations that Chung described in establishing (9) and (10).

\section{Long-range dependence of functionals}

Returning to the general process $\left\{Y_{n}\right\}$ for which (2) holds, we can write for (1),

$$
\begin{aligned}
\operatorname{var}\left(Y_{0}+\cdots+Y_{n-1}\right)-n \operatorname{var}\left(Y_{0}\right) & =2 \sum_{r=1}^{n-1}(n-r) \operatorname{cov}\left(Y_{0}, Y_{r}\right) \\
& =2 \sum_{i} \sum_{j} y_{i} y_{j} \pi_{i} \sum_{r=1}^{n-1}(n-r)\left(p_{i j}^{r}-\pi_{j}\right) \\
& =2 \sum_{i} \sum_{j} y_{i} y_{j} \pi_{i} R_{i j}^{n-1},
\end{aligned}
$$

where, as for (6),

$$
R_{i j}^{n}=Q_{i j}^{1}+\cdots+Q_{i j}^{n} .
$$

Formally then, because Lemma 3 gives $R_{i j}^{n} \sim\left(\pi_{j} / \pi_{k}\right) R_{k k}^{n}$ for $n \rightarrow \infty$, we can write

$$
\begin{aligned}
\frac{\operatorname{var}\left(Y_{0}+\cdots+Y_{n}\right)-(n+1) \operatorname{var}\left(Y_{0}\right)}{2 R_{k k}^{n} / \pi_{k}} & =\sum_{i} \sum_{j} y_{i} y_{j} \pi_{i} \pi_{j} \frac{R_{i j}^{n} / \pi_{j}}{R_{k k}^{n} / \pi_{k}} \\
& \rightarrow \sum_{i} \sum_{j} y_{i} y_{j} \pi_{i} \pi_{j},
\end{aligned}
$$

where interchange of the summation operations on $i$ and $j$ with the limit operation on $n$ is justified if there are only finitely many nonzero summands in $i$ (and $j$ ).

Since $R_{k k}^{n} / n \rightarrow \infty$, the term $(n+1) \operatorname{var}\left(Y_{0}\right)$ can be omitted from the left-hand side of (13) without affecting any limit (when it exists). Observe that applying the lim sup version of Fatou's lemma with respect to the summation on $i$ and $j$ in (13), we do have

$$
0 \leq \limsup _{n \rightarrow \infty} \frac{\operatorname{var}\left(Y_{0}+\cdots+Y_{n}\right)}{2 R_{k k}^{n} / \pi_{k}} \leq \sum_{i} \sum_{j} y_{i} y_{j} \pi_{i} \pi_{j} \cdot 1=\left(\sum_{i \in \mathcal{X}} y_{i} \pi_{i}\right)^{2} .
$$

Thus, for a square integrable functional $Y_{n}$ defined on a stationary, long-range dependent Markov chain, the rate of growth of $\operatorname{var}\left(Y_{1}+\cdots+Y_{n}\right)$ is at most proportional to the rate of growth of $\operatorname{var}\left(N_{k}(0, n]\right)$ for any state $k$. 
Let $A$ be a proper subset of $\mathcal{X}$, and write $\left\{I_{n A}\right\}$ for the stationary indicator process for $\left\{X_{n} \in A\right\}$ and $N_{A}(0, n]=\sum_{r=1}^{n} I_{r A}$. Then when $A$ is finite, the argument just given proves that $\left\{I_{n A}\right\}$ is long-range dependent with growth behaviour of the covariance terms proportional to that of $R_{k k}^{n}$ for any state $k$. Furthermore, since, for the set $A^{\mathrm{c}}$, the complement in $\mathcal{X}$ of $A$, we have

$$
\operatorname{cov}\left(I_{0 A^{\mathrm{c}}}, I_{n A^{\mathrm{c}}}\right)=\operatorname{cov}\left(I_{0 A^{\mathrm{c}}}, 1-I_{n A}\right)=-\operatorname{cov}\left(I_{0 A^{\mathrm{c}}}, I_{n A}\right)=\operatorname{cov}\left(I_{0 A}, I_{n A}\right),
$$

we can now conclude that $\operatorname{var}\left(N_{A}(0, n]\right)$ and $\operatorname{var}\left(N_{A^{\mathrm{c}}}(0, n]\right)$ have the asymptotic behaviour of $R_{k k}^{n}$ whenever $A$ or its complement is a nonempty finite set. Specifically, with $\pi_{A}=\sum_{i \in A} \pi_{i}$,

$$
\frac{\operatorname{var}\left(N_{A}(0, n]\right)-n \pi_{A}\left(1-\pi_{A}\right)}{2 R_{k k}^{n} / \pi_{k}} \rightarrow\left(\pi_{A}\right)^{2} \quad \text { as } n \rightarrow \infty .
$$

A similar argument yields the (slight) generalisation implicit in (14) of Proposition 1, below. The Hurst index property is a consequence of definition and the class property given below (11).

Proposition 1. When $Y_{n}=y_{X_{n}}$ is a functional of the long-range dependent Markov chain $X_{n}$ and $y_{i} \neq 0$ on only a finite subset $A$ of the state space, then

$$
\frac{\operatorname{var}\left(Y_{1}+\cdots+Y_{n}\right)}{2 n Q_{k k}^{n}} \rightarrow \frac{\left(\sum_{i: y_{i}>0} y_{i} \pi_{i}\right)^{2}}{\pi_{k}} \text { as } n \rightarrow \infty,
$$

and $\left\{Y_{n}\right\}$ has the same Hurst index, (11), as the chain $\left\{X_{n}\right\}$.

\section{Some examples}

Example 1. Theorem 11.2 of Chung (1967), based on 'repetitive patterns' in his Section 8 or else Examples XV.2(k) and (1) of Feller (1968), exhibits an example of an irreducible Markov chain on the nonnegative integers $\{0,1, \ldots\}$ which, with a suitable choice of parameters, can be stationary with the moment index of the return times taking any prescribed value $\kappa \geq 1$, and thus the chain is long-range dependent when $\kappa<2$ (and also when $\kappa=2$ but $m_{00}^{(2)}=\infty$ ), with Hurst index $\frac{1}{2}(3-\kappa)$. These examples were also presented by Meyn and Tweedie (1993, pp. 43-44) as special cases of the forward and backward recurrence time chains, respectively.

Example 2. In a stable single-server queueing system with Poisson arrivals at rate $\lambda$ and independent, identically distributed (i.i.d.) service times $\left\{S_{n}\right\}$ with mean $\mathrm{E}\left(S_{n}\right)<1 / \lambda$ and moment index $\kappa_{S}$ lying in $(3,4)$, Carpio (2006) showed that if the sequence of serial correlation coefficients $\left\{r_{n}\right\}$ of the stationary process of queue sizes at departure epochs $\left\{X_{n}\right\}$ behaves asymptotically as $c n^{-\alpha}$ for some finite positive $c$ and $\alpha \in(0,1)$, where $\alpha=\kappa_{S}-3$, then $\left\{X_{n}\right\}$ has Hurst index $\frac{1}{2}\left(5-\kappa_{S}\right)$, i.e.

$$
\sup \left\{h: \limsup _{n \rightarrow \infty} \frac{\operatorname{var}\left(X_{1}+\cdots+X_{n}\right)}{n^{2 h}}=\infty\right\}=\frac{5-\kappa_{S}}{2} .
$$

When $\left\{X_{n}\right\}$ is long-range dependent with $\mathrm{E}\left(S_{n}^{r}\right)<\infty$ for a given $r \in(3,4), r_{n}$ satisfies $\lim \sup _{n \rightarrow \infty} n^{r-3} r_{n}<\infty$. The finiteness of the second moment of the first return times to the state 0 of the Markov chain $\left\{X_{n}\right\}$ was crucial in establishing this relationship; its finiteness is dependent on the finiteness of the second moment of $X_{n}$. The same Hurst index for the long-range dependent properties of the Markov chain $\left\{X_{n}\right\}$ arises as for the critical index $h$ 
in (15). The chain $\left\{X_{n}\right\}$ is a stochastically monotone Markov chain which means that, for a fixed state $j$ and every pair of states $i$ and $k$, where $i<k$,

$$
\mathrm{P}\left(X_{n+1} \leq j \mid X_{n}=i\right) \geq \mathrm{P}\left(X_{n+1} \leq j \mid X_{n}=k\right) .
$$

Example 3. Heyde (1988) noted that the stationary process of waiting times $\left\{W_{n}\right\}$ in a singleserver queue with arrivals at epochs of a renewal process with generic lifetime RV $T$ with finite mean $\mathrm{E}(T)$ larger than a generic element of the sequence of independent service times $\left\{S_{n}\right\}$ as in Example 2, constitutes a long-range dependent process when the moment index of service times $\kappa_{S}$ lies in $(3,4)$, again as in Example 2. For such a chain, the sequence of indicators $\left\{I_{W_{n}=0}\right\}$ is a renewal process, which is shown to be long-range dependent, as in Section 2, precisely when $\kappa_{S} \in(3,4)$. Similar to Example 2, Carpio (2006) showed that if the sequence of serial correlation coefficients $\left\{\rho_{n}\right\}$ of the stationary process $\left\{W_{n}\right\}$ behaves asymptotically as $c n^{-\alpha}$ for some finite positive $c$ and $\alpha \in(0,1)$, where $\alpha=\kappa_{S}-3$, then $\left\{W_{n}\right\}$ has Hurst index $\frac{1}{2}\left(5-\kappa_{S}\right)$. When the tail of the service time distribution function is regularly varying, the long-range dependent stationary process $\left\{W_{n}\right\}$ also has Hurst index $\frac{1}{2}\left(5-\kappa_{S}\right)$.

\section{A conjecture and two counter-examples}

Examples 2 and 3 lend weight to the conjecture that when a stationary Markov chain $\left\{X_{n}\right\}$ is long-range dependent, any nontrivial functional $\left\{Y_{n}\right\} \equiv\left\{y_{X_{n}}\right\}$ of the chain (meaning, that $\operatorname{var}\left(Y_{n}\right)$ is finite and positive) is long-range dependent with the same Hurst index. This property is borne out by a simulation study of the two components of a stationary work-load vector in a 2-server queue of type $\mathrm{M} / \mathrm{P} / 2$, where $\mathrm{P}$ denotes i.i.d. service times having a Pareto distribution with $\kappa_{S}=\frac{7}{2}$, irrespective of the system being stable or unstable as a single-server system. When such a system is stable as a single-server system, so that its mean interarrival time exceeds its mean service time, the moment index of the mean delay exceeds the moment index of the larger component of the Kiefer-Wolfowitz work-load vector; see Scheller-Wolf (2003). Carpio (2006, Chapter 5) applied various empirical estimators to find the Hurst index of the waiting times and queue sizes at departure epochs of M/P/2 queues and M/P/1 queues. Under a given empirical estimator, the Hurst parameter estimates from both queues were taken for a given pair of the traffic intensity $\rho=\mathrm{E}\left(S_{n}\right) / s \mathrm{E}(T)$, where $s$ denotes the number of servers, and $\kappa_{S}$. The outcome shows that the resulting Hurst parameter estimates are almost identical.

However, the following two examples show that the conjecture is false, and instead point to the problem of identifying what nontrivial functionals of what Markov chains do remain long-range dependent and with what Hurst index.

Example 4. This example, relying on the work of Daley (2007) (with a sequel in Daley et al. (2007)), entails a Markov chain $\left\{X_{n}\right\}$ on the nonzero positive and negative integers as state space. Whenever the chain visits +1 or -1 it jumps to state $-n$ or $+n$, respectively, with probability distributions $\left\{g_{-n}\right\}$ and $\left\{f_{n}\right\}$, respectively, while, for integers $n \geq 2$, from $+n$ it moves to $+(n-1)$ and from $-n$ it moves to $-(n-1)$. The probability distributions $\left\{g_{-n}\right\}$ and $\left\{f_{n}\right\}$ have finite first moments but infinite second moments. Such a chain has a simpler interpretation as an alternating renewal process, but for the purpose of this paper it is better formalised as an irreducible Markov chain on a countable state space. For the functional $\left\{Y_{n}\right\}$, defined via $y_{i}=1$ if and only if $i$ is a positive integer, $S_{n}=\sum_{r=1}^{n} Y_{r}$ is the amount of time that $X_{n}$ spends in the positive integers. Then, as shown by Daley $(2007), \operatorname{var}\left(S_{n}\right) / \operatorname{var}\left(N_{1}(0, n]\right)$ can oscillate indefinitely, and more wildly, as $n$ tends to $\infty$, when the ratio of the truncated 
second moments $\sum_{r=1}^{\infty} \min (r, n) \sum_{s>r} f_{s}$ and $\sum_{r=1}^{\infty} \min (r, n) \sum_{s>r} g_{-s}$ does not converge for $n$ tending to $\infty$.

Example 5. This example is due to the referee. Consider two positive recurrent, aperiodic, irreducible Markov chains, $\left\{X_{n}^{\prime}\right\}$ and $\left\{X_{n}^{\prime \prime}\right\}$ say, on the nonnegative integers. Let the return time to a fixed state have infinite second moment in $\left\{X_{n}^{\prime}\right\}$ and finite second moment in $\left\{X_{n}^{\prime \prime}\right\}$. Let $\left\{X_{n}\right\}=\left\{\left(X_{n}^{\prime}, X_{n}^{\prime \prime}\right)\right\}$ be the chain on the product space with the components evolving independently of each other. Trivially, the chain $\left\{X_{n}\right\}$ is long-range dependent as described in Sections 1 and 2 above. For the functional $Y_{n}^{\prime}$, say, which is equal to the indicator of a fixed state for $\left\{X_{n}^{\prime}\right\},\left\{Y_{n}^{\prime}\right\}$ is long-range dependent, while for the functional $Y_{n}^{\prime \prime}$, say, which is equal to the indicator of a fixed state for $\left\{X_{n}^{\prime \prime}\right\},\left\{Y_{n}^{\prime \prime}\right\}$ is not long-range dependent.

\section{Acknowledgements}

We thank an anonymous referee for comments, especially those which led to the addition of Example 5. Part of this work was carried out while visiting the Institut Mittag-Leffler, Stockholm.

\section{References}

CARPIO, K. J. E. (2006). Long-range dependence of Markov chains. Doctoral Thesis, The Australian National University. Chung, K. L. (1967). Markov Chains with Stationary Transition Probabilities, 2nd edn. Springer, New York.

DALEY, D. J. (1968). Stochastically monotone Markov chains. Z. Wahrscheinlichkeitsth. 10, 305-317.

DAley, D. J. (1978). Upper bounds for the renewal function via Fourier methods. Ann. Prob. 6, 876-884.

Daley, D. J. (1999). The Hurst index of long-range dependent renewal processes. Ann. Prob. 27, 2035-2041.

Daley, D. J. (2001). The moment index of minima. In Probability, Statistics and Seismology (J. Appl. Prob. Spec. Vol. 38A), Applied Probability Trust, Sheffield, pp. 33-36.

DALEY, D. J. (2007). Long-range dependence in a Cox process directed by an alternating renewal process. Submitted.

Daley, D. J. And Vere-Jones, D. (2003). An Introduction to the Theory of Point Processes, Vol. 1, 2nd edn., Springer, New York.

Daley, D. J. AND Vesilo, R. (1997). Long range dependence of point processes, with queueing examples. Stoch. Process. Appl. 70, 265-282.

Daley, D. J., RolsKi, T. And Vesilo, R. (2007). Long-range dependence in a Cox process directed by a Markov renewal process. To appear in J. Appl. Math. Decision Sci.

FELLER, W. (1966). On the Fourier representation for Markov chains and the strong ratio theorem. J. Math. Mech. 15, 274-283.

Feller, W. (1968). An Introduction to Probability Theory and Its Applications, Vol. 1, 3rd edn. John Wiley, New York.

HeYde, C. C. (1988). Asymptotic efficiency results for the method of moments with application to estimation for queueing processes. In Queueing Theory and Its Applications, eds O. J. Boxma and R. Syski, North-Holland, Amsterdam, pp. 405-412.

Meyn, S. P. And Tweedie, R. L. (1993). Markov Chains and Stochastic Stability. Springer, London.

Orey, S. (1961). Sums arising in the theory of Markov chains. Proc. Amer. Math. Soc. 12, 847-856.

SCHELler-Wolf, A. (2003). Necessary and sufficient conditions for delay moments in FIFO multiserver queues with an application comparing $s$ slow servers with one fast one. Operat. Res. 51, 748-758.

Sgibnev, M. S. (1981). Renewal theorem in the case of infinite variance. Siberian Math. J. 22, 787-796.

Sgibnev, M. S. (1996). An infinite variance solidarity theorem for Markov renewal functions. J. Appl. Prob. 33, 434-438. 